\title{
PATOLOGÍAS DEL IDEAL: VARIEDADES CLÍNICAS DE LA DENOMINADA “DISTORSIÓN PERCEPTIVA" *
}

\author{
EGO IDEAL'S PATHOLOGIES: CLINICAL VARIETIES OF SO-CALLED \\ "PERCEPTUAL DISTORTION".
}

Fecha de recepción: 07-2-2017 Fecha de aceptación: 19-3-2017

LUIS VOLTA

Lic. en Psicología. Psicólogo de Planta e Instructor de Residentes de Psicología del HIGA Prof. Dr. R. Rossi. Docente - Investigador de grado y posgrado de la Facultad de Psicología Universidad Nacional de La Plata (UNLP) y Universidad Católica de La Plata (UCALP).

Resumen: En el presente trabajo nos proponemos indagar la función del Ideal del Yo en el condicionamiento de la aparición de la denominada "distorsión perceptiva”. Dicho fenómeno clínico, de fundamental importancia en la caracterización de cuadros clínicos tales como la anorexia, la obesidad, la vigorexia y la dismorfia merece un esclarecimiento en términos de clínica diferencial. Sólo despejando su inserción en la estructura del sujeto y la diversidad de funciones que allí cumple podrán diseñarse estrategias terapéuticas adecuadas en la interfase psicoanálisis-salud mental.

Palabras Clave: Ideal del yo - Distorsión perceptiva Diagnóstico Diferencial - Dismorfia
Abstract: This work is aimed at inquire Ego Ideal's function conditioning the so-called perceptual distortion. This clinical phenomenon, so important in anorexy, obesity, bigorexia, and dysmorphia descriptions should be clarified in differential diagnosis. Only by clearing it's insertion and function in subject's structure we will be able to design adequate therapeutic strategies in the psychoanalysis-mental health interface.

Key Words: Ego Ideal - Perceptual distortion - Differential diagnosis - Dysmorphia

"Pues quitadlo de allí, y el hombre no puede ya ni siquiera sostenerse en la posición de Narciso" Jacques Lacan 1957-1958

LA PERCEPCIÓN EN PSICOANÁLISIS: EL CASO PARTICULAR DEL CUERPO

Con "La perturbación psicógena de la visión según el psicoanálisis" (1910) y "Formulaciones sobre los dos principios del acaecer psíquico" (1911) Sigmund Freud sentó las bases para una teoría de la percepción propiamente psicoanalítica, contraria a cualquier aprehensión empirista ingenua, o centrada exclusivamente en el carácter sensorial de nuestra relación con el mundo. La realidad para un sujeto, a pesar de que pueda parecerle que se presenta a través de una simple intuición directa, es en realidad el efecto de la intervención de pulsiones y representaciones que en su entramado evitan lo insoportable y dan carácter de evidencia a lo percibido.

Por su parte Jacques Lacan pudo distinguir los registros que intervienen en dicho condicionamien- to. En primer lugar, con su conceptualización del Estadio del Espejo (1949) cuando afirma que "la imagen especular parece ser el umbral del mundo visible" (1). En segundo término, con su célebre crítica de la teoría clásica de la alucinación como Perceptum sin objeto (1958), al demostrar el carácter determinado del sujeto y de su percepción por su dependencia del orden simbólico. Esto le permite situar la importancia del Ideal del yo, como "posición I desde donde percibe" (2) y que condiciona la buena forma del Yo ideal. Finalmente, introduce el objeto $a$ como real fuera de la imagen y del sentido, disyunto de la "realidad" organizada en términos de fantasma, pero para la que constituye una amenaza angustiosa. Algunos años más tarde podrá sintetizar todos estos argumentos afirmando: "No hay la más mínima realidad prediscursiva" (3). Si la realidad deviene 
del discurso, es el significante amo, S1, parte fundamental del mismo la escritura que condensa la potencia ordenadora del Ideal en el que el sujeto se hipostasía.

La percepción del cuerpo no es ajena a este planteo. Así, y tal como lo condensa en una fórmula C. Soler (1983) "el cuerpo es una realidad" (4). Su percepción no puede nunca asimilarse a un dato primario. En consecuencia, cuando una anoréxica nos dice que no come porque "se ve gorda"; o por el contrario, cuando un obeso come de más "porque no se ve mal", cuando un joven físicoculturista no para de entrenar porque "ve que se achica"; o cuando un sujeto demanda insistentemente una cirugía estética porque "se ve fea/o", una pregunta se le puede plantear a quien lo escucha: ¿Desde dónde se mira? La misma, como veremos, resulta ser fecunda al intentar despejar las amarras que sostienen la consistencia estética de la imagen del cuerpo.

En el presente trabajo nos proponemos entonces indagar la función del Ideal del Yo en el condicionamiento de la aparición de la denominada "distorsión perceptiva”. Dicho fenómeno clínico, de fundamental importancia en la caracterización de cuadros tales como la anorexia, la obesidad, la vigorexia y la dismorfia merece un esclarecimiento en términos de clínica diferencial. Sólo despejando su inserción en la estructura del sujeto y la diversidad de funciones que allí cumple podrán diseñarse estrategias terapéuticas adecuadas en la interfase psicoanálisis-salud mental.

\section{DISTORSIONES PERCEPTIVAS Y CLÍNICA DIFERENCIAL NEUROSIS - PSICOSIS}

En continuidad con la problemática esbozada, cabe interrogarnos sobre el momento inaugural en la constitución subjetiva y la conformación del yo en torno a la imagen especular en las Neurosis y en las Psicosis, para pensar las variedades clínicas de la distorsión perceptiva desde la perspectiva de las patologías del Ideal.

En el caso de las neurosis, para que la imagen que el sujeto ve en el espejo aparezca como "una" y adquiera consistencia será necesaria la identificación con una constelación de significantes privilegiados tomados del Otro: I (A); insignias que lo constituyen y alienan en esa identificación primera que forma el Ideal del yo. Al mismo tiempo, la identificación al Ideal es correlativa de la simbolización del deseo por el significante fálico; lo que haría posible la función de corte por la que puede caer el objeto $a$. Así, los momentos de vacilación del Ideal encuentran su correlato clínico en la emergencia de la señal de angustia, llegando en algunos casos más extremos hasta la despersonalización,

En la psicosis, el Ideal del yo no opera de la misma manera que en la neurosis donde organiza el campo perceptivo junto al fantasma, enmarcando al objeto. Aquí más bien, el sujeto se ve en ocasiones confrontado a la presencia de un objeto no extraído. "Io sono sempre vista", decía la paciente de Bobon testimoniando de la invasión constante de la mirada, objeto no negativizado por la castración. "En oposición a la mirada que pasa a lo real, la función del Ideal del Yo, la gran I de la neurosis, es una sublimación de esta mirada. En la perspectiva en la que se admite la igualdad clínica de los parlêtres, la mirada está en lo más profundo en lo real y la neurosis permite situarla en el registro del ideal" (5). En función de lo expuesto, presentamos tres casos que ilustran la diversidad clínica del fenómeno.

\section{ESTUDIO DE CASO 1}

Tanto en sus dichos como en su imagen corporal $\mathrm{V}$., de 26 años, refleja un ideal de perfección: ser el alumno, el novio, el amante perfecto... trabaja para lograr eso. Sin embargo, uno de los motivos que lo trae a la consulta es su sensación de inseguridad y su preocupación en exceso por cómo lo ven los demás. Síntomas que estaban "tapados" y retornaron luego de un episodio que nombra como "traumático". En una fiesta electrónica dice haber excedido sus límites y termina bailando desnudo, haciendo gestos agresivos a la gente: "eran como siluetas, fantasmas... de sentirme observado por esa gente y era como decirles que no me importaba nada". Luego de que se viralizara un video donde $\mathrm{V}$. queda expuesto, comienza a sentir que sus compañeros, profesores y amigos lo miran, hablan de él, lo señalan con el dedo, muy atento a cualquier comentario sobre lo sucedido, y con el temor a ser juzgado por eso. Se trató de una exhibición del sexo en completa contradicción con lo que habitualmente cree dar a ver de sí, - un yo inflado de proezas y hazañas - y que lo sumerge en muchas preguntas. Su virilidad misma aparece cuestionada, en tanto que en la fiesta hacía gestos que podían llegar a parecer homosexuales. Posteriormente, al buscar demostrar su hombría teniendo sexo, lo interrogan situaciones de impotencia y de pérdida de deseo sexual por una mujer de mala reputación. Cree que otros sí podrían satisfacerla.

Este episodio y la conmoción que le generó, lo lleva a V. a interrogar un síntoma que lo acompaña 
desde su infancia: "sentirse observado", con un correlato de angustia que lo conducía a encerrarse y aislarse. Nombra a esto "inseguridad" frente a la pregunta que insiste: “ ¿cómo me ven los demás?”. En el colegio, sentirse mirado y juzgado lo llevó a tener dificultades en el encuentro con pares, no podía mirar a los ojos a las personas, desviaba la mirada. A sus 15 años, su padecimiento se agudiza y busca tomar medidas drásticas. Luego de un mes de encierro, consulta con un cirujano para operarse los ojos, en particular los párpados en los que denuncia un rasgo "oriental". Afirma, "no los veía como los de los demás”, lo que ubica en ese momento como la causa de no ser aceptado y de no estar "a la altura". Compararse con los demás lo llevaba a cuestionar su aspecto físico, sumado a las "cargadas" de sus compañeros: "feo", "flaco", "chino", "orejón". Cambiar sus ojos, luego sus orejas, finalmente su nariz como estrategia para acceder a aquel goce que le estaría vedado pero accesible a los otros: "operarme para poder vivir lo que vivían los demás." Aquellos otros que describe con confianza y seguridad, que disfrutaban de salidas y tenían novias, todo aquello para lo cual él no se sentía "capaz". Un tratamiento psicológico y psiquiátrico oportuno evita llevar a cabo las operaciones. Concluye, sin embargo: "Siempre busco la aprobación de los demás."

Si bien los síntomas desaparecieron por algún tiempo esto no derivó en un verdadero cambio de posición. Ubica el fallecimiento de su madre, de quien sacó sus ojos y desde cuya mirada se orienta, como lo que le dio el empuje para realizar distintas actividades, haciendo "todo por ella" y buscando que "a todo el mundo le guste": cocina, guitarra, gimnasio. Sitúa que, a partir de modificar notablemente su imagen corporal con el entrenamiento físico, cambió su relación con los otros, volviéndose más sociable, y la paradoja de sentirse menos observado. Frente al actual retorno del síntoma, se debate, entre intentar reconstruir la imagen de perfección perdida, o por primera vez, y en las coordenadas de un tratamiento, "dejar de conformar a los demás" a partir de revisar su posición frente aquello que en el sexo hace agujero y que ninguna imagen puede colmar. Empresa particularmente difícil, tal como lo advierte Lacan, "alejar al obsesivo del dominio de la mirada" (6).

\section{ESTUDIO DE CASO 2}

El punto de mayor padecimiento de R. (43 años) yace en la mirada del Otro que le retorna en lo real con fenómenos concomitantes a nivel del cuerpo y del pensamiento: la experiencia de ser mirado se acompaña de sudoración, temblores, cefaleas y la idea certera de que lo miran para burlarse de él. Si además los ve hablar o reírse, interpreta que esos dichos y esas risas le conciernen: “¿has visto cómo está vestido? ¿Por qué es tan bajito? a esta persona no se le ocurre hablar, es tan raro". Hablan de su imagen, de este "bicho raro"; expresión con la que se nombra descontándose del conjunto de los "normales". Demanda al tratamiento una solución que lo incluya dentro del parámetro de una normalidad supuesta frente a lo enigmático que se le torna el lazo con los otros y su propio cuerpo. Aquello que R. interpreta a partir de la mirada del Otro redobla su fuerte rechazo a mirarse en el espejo. Está disconforme con lo que éste refleja. Se siente "poco agraciado": ojos hundidos, muy delgado, poca estatura, nariz muy gruesa, y una cicatriz en la mandíbula que le recuerda el accidente en el que murió su padre cuando era pequeño; acontecimiento que ubica como respuesta a la pregunta por el inicio de su padecimiento, como si dicha pérdida hubiese privado a este sujeto, de la orientación por las insignias paternas.

En este caso, el espejo no funciona como un recurso que resguarde al sujeto del temor obsesivo de la propia deformidad corporal. No hay aquí amor propio, ni adoración del cuerpo como principio de la imaginación. Por el contrario, el espejo es un elemento que el paciente evita puesto que lo enfrenta a una verdadera ausencia de cobertura, y lo expone a una experiencia que delata una falla a nivel de la constitución de lo imaginario. ¿Cuál es el Ideal desde el cual se mira R.? En lo que atañe a la imagen, si bien ha contemplado la posibilidad de practicarse una cirugía estética sabe que ello no solucionaría su problema. La comparación con los otros y la experiencia de sentirse en menos, no se limita a la cuestión de la imagen corporal, sino que atañe otras coordenadas vinculadas a lo supuestamente esperable para un hombre de su edad: ser un profesional, tener un trabajo estable, haber formado una familia. Ideales tomados del padre que sus hermanos han podido concretar y él no. Sin embargo, a diferencia de ellos y de muchos otros, R. cuenta con una habilidad singular que comienza a practicar luego de la muerte del padre: puede reparar objetos rotos que otros dan por perdidos. Nombrarse "reparador" constituye un significante alternativo que le permite, por momentos, incluirse como uno entre otros, y despegarse de la identificación al objeto resto, nombrada por R. como "baja autoestima". Sin 
embargo, esto resulta ser una solución precaria e insuficiente para devolverle una consistencia estética a su imagen.

\section{ESTUDIO DE CASO 3}

D. (32 años) se presenta aquejado de un malestar que nombra espontáneamente como "fobia social" describiendo la ansiedad y miedo que le generan las situaciones sociales. Este padecimiento ya es "muy difícil de controlar", debido al inicio de sus estudios terciarios en donde debe compartir clases junto a muchas personas.

A lo largo de las entrevistas se irá ubicando la presencia de este malestar desde su adolescencia, bajo la forma de cierta inhibición social y una dificultad para tomar la palabra al interactuar con los otros. Esto lo llevaba recurrentemente a evitar las salidas de su casa, logrando paliarlo desde hace unos años acoplándose a la figura de su pareja, quien devino su principal referencia para desenvolverse en el mundo. Es éste quien lo acompaña a todos lados, funcionando como una suerte de ideal exterior. Su partenaire sabe cómo desenvolverse y qué decir en cada ocasión, así como también lo orienta sobre qué decisiones tomar, incluso cómo vestirse.

Este frágil equilibrio sostenido desde entonces comienza a vacilar a partir de lo que D. nombra como una "sensación rara" en el ojo, "como una basurita, la sensación de ojo seco, como que me titila" que le hace especialmente incómodo el vínculo con otros. Podrían mirarle su ojo. El gesto de cualquier persona que se toque su propio ojo desencadena en él una idea cerrada a toda composición dialéctica: lo hacen para burlarse. Llegará a una de las últimas entrevistas muy angustiado relatando una experiencia paranoide en donde la certeza de ser mirado aparece en forma radical: "notaba que todos miraban mi ojo, se burlaban y se reían de mí, me daba cuenta porque se tocaban el ojo". Se trata de una mirada invasora que desarma la consistencia del cuerpo. D. nos relata cómo intentaba negativizar esa experiencia deslocalizada: "cuando se burlaban me tocaba fuerte el ojo para empujarlo para adentro, se me salía... pero no sé bien cuál de los dos ojos es". Razón que lo lleva a recurrir al discurso médico buscando allí una solución a este "defecto" que se le presenta como la causa de su tormento. Llamado exitoso, en este primer momento, en tanto encuentra en el diagnóstico de una patología paranasal, no solo un nombre que lo tranquiliza sino la esperanza de una curación posible. Aquí el Ideal, lejos de constituir una parte del yo diferenciada por el atravesamiento del complejo de Edipo y efecto de los movimientos de inscripción simbólica, constituye más bien una instancia encarnada en una figura externa, que le permite aliviar la mirada, objeto que también proviene de lo real.

Recuerda haberse dado cuenta de su "defecto" a partir de una escena en la que escucha a su suegra burlarse de él a sus espaldas. Ella decía "guarda que se te va a caer el ojo", "nos vamos a tener que alejar de la pantalla para que no se nos agrande el ojo"... "yo ahí empecé a pensar y me di cuenta que era por el tema de mi ojo que se me había sobresalido". Dicha escena es contemporánea a la crítica recibida desde sus hermanas respecto de su posición de "sumisión" en la pareja, cuestionamiento de la solución hasta el momento encontrada para su fragilidad enunciativa.

\section{DISCUSIÓN Y CONCLUSIONES}

El recorrido realizado nos ha permitido constatar la importancia de la función del Ideal del yo en el armado de la consistencia estética de la imagen del cuerpo. En los casos analizados, y en coincidencia con otros casos reportados por la bibliografía sobre el tema, la buena forma no logra precipitarse y los sujetos apelan a diversos recursos para hacer frente al problema perceptivo. En este sentido, nos ha parecido importante no considerar la aparición de los diversos episodios como meras irrupciones aisladas de una misma perturbación perceptiva que deba ser normalizada. No se trata de desarticularlos del resto de la vida de los sujetos, sino que es necesario inscribirlos en una organización más amplia en la que se conjuguen contextos históricos y condicionantes subjetivos que permitan aprehenderlos en una lógica singular.

Es de destacar que la coyuntura de aparición de la llamada "distorsión perceptiva", coincide en varios de ellos con las vacilaciones y reconfiguraciones del Ideal del yo, propias de la adolescencia (Casos 1 y 3), momento en que el sujeto debe poner a prueba sus recursos simbólicos y enfrentarse con lo que en la sexualidad "hace agujero en lo real". (7)

Al mismo tiempo, aquellos casos que se esclarecen apelando a la lógica y funcionamiento propios de la estructura de la psicosis, se presentan más bien en términos de continuidad y de desenganches, que de francos desencadenamientos procesuales clásicos. Esta cuestión nos pone sobre la pista de la importancia del fenómeno estudiado, en los casos en que el trabajo del sujeto se apoya preponderantemente en el registro imaginario.

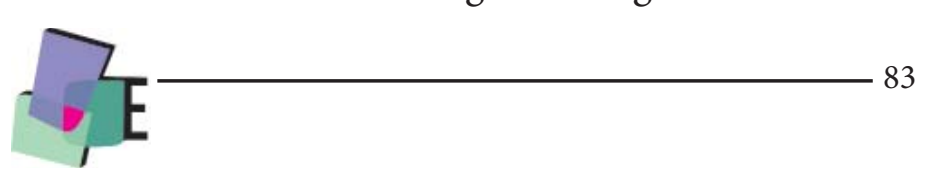


Por estos motivos, nos ha resultado importante en cada caso, zanjar la cuestión de si se trata de una apelación al imaginario especular como forma de remediar la ausencia de significación fálica o como una manifestación de la castración a nivel del yo $(-\varphi)$.

En todo caso, la mejoría clínica reconocible en algunos de ellos, lejos de efectivizarse a partir de intervenciones médico-quirúrgicas, estuvo comandada por una modificación de la posición del sujeto en lo simbólico, correlativa a una transformación de su posición libidinal.

Esta última constatación, invita a llevar adelante un trabajo en conjunto con aquellas especialidades médicas que suelen recibir las demandas de tales pacientes, para prevenir intervenciones estériles o demasiado pesadas en sus consecuencias. Si bien una de las consecuencias mayores de la caída de los grandes relatos en la posmodernidad, ha sido la del ascenso del valor de la imagen del cuerpo y la pretensión de reducción del sujeto al organismo, debemos permanecer advertidos del "misterio del cuerpo que habla" (8). Éste está incurablemente sometido a una experiencia de goce sin imagen que "se presenta al mismo tiempo como presencia de Otra cosa, y como ausencia de una instancia de percepción y representación que pueda corresponder a ella" (9).

\section{NOTAS}

* Trabajo presentado por Luis Volta en coautoría con Residentes de Psicología del Servicio de Salud Mental del HIGA Prof. Dr. R. Rossi de La Plata: Andrea Lopez Bonanni, Juan Manuel Blanco, Lucía Estévez, Pilar Loza Colomer, Paula Marchesi, Flavia Iacusso, Ana Paula Ribeiro, Laura González Elizaga, Gisele Dubles y Mónica Gasparín.

(1) Lacan, J.: "El estadio del espejo como formador de la función del yo (je) tal como se nos revela en la experiencia psicoanalítica", en Escritos 1, Siglo XXI Editores, Buenos Aires, 1987, pág. 88.

(2) Lacan, J.: "Observación sobre el informe de Daniel Lagache: "Psicoanálisis y Estructura de la Personalidad”, en Escritos 2, Siglo XXI Editores, Buenos Aires, 1987, pág. 659.

(3) Lacan, J.: El Seminario, libro 17, El Reverso del Psicoanálisis, (19691970), Paidós, Buenos Aires, 1996, pág. 44.

(4) Soler, C: "El cuerpo en la enseñanza de Jacques Lacan", en Estudios de psicosomática Vol. I, recopilación Vera Gorali, Ed. Atuel, Buenos Aires. 1993, pág. 94 .

(5) Laurent, E.: El reverso de la biopolítica, Grama, Buenos Aires, 2016, p. 228.

(6) Lacan, J.: El Seminario, libro 23, El Sinthome (1975-1976), Paidós, Buenos Aires, 2006, pág. 18.

(7) Lacan, J.: "Prefacio al Despertar de la Primavera", en Otros escritos, Paidós, Buenos Aires, 2012, p. 588.

(8) Lacan, J.: El Seminario, libro 20, Aún (1972-1973), Paidós, Buenos Aires, 1996, pág. 158.

(9) Laurent, E.: El reverso de la biopolítica, Grama, Buenos Aires, 2016, p. 17

\section{BIBLIOGRAFÍA}

-De Preester, H., Knockaert, V.: Body Image and Body Schema: Interdisciplinary Perspectives on the Body, John Benjamins Publishing, Amsterdam, 2005.

-Freud, S.: "La perturbación psicógena de la visión según el psicoanálisis" (1910), AE, XI, Buenos Aires, 1991, pp: 205-216.

-Freud, S.: "Formulaciones sobre los dos principios del acaecer psíquico" (1911), AE, XII, Buenos Aires, 1991, pp: 217-232.

-Halmi KA, Goldberg SC \& Cunningham S.: "Perceptual distortion of body image in adolescent girls: distortion of body image in adolescence", en Revista Psychological Medicine, V. 7(2), 1977:253-7.

-Hennighausen K: Enkelmann, D. Wewetzer, C. \& Remschmidt, H.:

"Body image distortion in Anorexia Nervosa - is there really a perceptual deficit?", en Revista European Child \& Adolescent Psychiatry, V. 8(3), 1999, pp 200-206.

-Miller, J-A.: “La imagen reina”, en Elucidación de Lacan, EOL-Paidós, Buenos Aires, 1998, pp. 577-602.

-Miller, J-A.: El peso de los ideales, EOL-Paidós, Buenos Aires, 1999.

-Morselli, E.: "Dismorfofobia y tafefobia: dos formas de locura con ideas fijas no descriptas hasta la actualidad", en La imagen del cuerpo propio y sus perturbaciones, Editorial de la Campana, La Plata 2009, pp. 57- 66. -Tignol, J.: Les défauts physiques imaginaires, Odile Jacob, Paris, 2006. 